\title{
Correction to: Effectiveness of chlorthalidone/amiloride versus losartan in patients with stage I hypertension and diabetes mellitus: results from the PREVER-treatment randomized controlled trial
}

\author{
Flávio D. Fuchs ${ }^{1}$ - Luiz C. N. Scala ${ }^{2}$. José F. Vilela-Martin ${ }^{3}$ - Paul K. Whelton ${ }^{4}$. Carlos E. Poli-de-Figueiredo ${ }^{5}$. \\ Ricardo Pereira E Silva ${ }^{6} \cdot$ Miguel Gus $^{1}$. Luiz A. Bortolotto ${ }^{7}$. Fernanda M. Consolim-Colombo ${ }^{7} \cdot$ Rosane P. Schlatter $^{8}$. \\ Evandro J. Cesarino ${ }^{9} \cdot$ Iran Castro ${ }^{10}$. José A. Figueiredo Neto ${ }^{11} \cdot$ Hilton Chaves ${ }^{12}$. André A. Steffens ${ }^{13}$. \\ João G. Alves ${ }^{14}$. Andréa A. Brandão ${ }^{15}$. Marcos R. de Sousa ${ }^{16}$. Paulo C. Jardim ${ }^{17}$. Leila B. Moreira ${ }^{8}$. \\ Roberto S. Franco ${ }^{18}$. Marco M. Gomes ${ }^{19}$. Abrahão Afiune Neto ${ }^{20}$. Felipe C. Fuchs ${ }^{1}$. Dario C. Sobral Filho ${ }^{21}$. \\ Antônio C. Nóbrega ${ }^{22} \cdot$ Fernando Nobre $^{23}$. Otávio Berwanger ${ }^{24} \cdot$ Sandra C. Fuchs ${ }^{1}$ (])
}

Published online: 1 March 2021

(c) Springer-Verlag Italia S.r.l., part of Springer Nature 2021

\section{Correction to: Acta Diabetologica}

https://doi.org/10.1007/s00592-020-01611-8

Authors would like to correct the given name of the coauthor "Evandro J. Cesarino". The author group is updated with corrected version.

The original article can be found online at https://doi.org/10.1007/ s00592-020-01611-8

Sandra C. Fuchs

sfuchs@hcpa.edu.br

1 Division of Cardiology, Hospital de Clínicas de Porto Alegre, Universidade Federal do Rio Grande Do Sul, INCT PREVER, CPC, $5^{\circ}$. and Ramiro Barcelos, 2350, Porto Alegre, RS 90035-903, Brazil

2 Hospital Universitário Júlio Müller, Universidade Federal de Mato Grosso, Cuiabá, MT, Brazil

3 Faculdade de Medicina São José Do Rio Preto, São José do Rio Preto, SP, Brazil

4 Department of Epidemiology, School of Public Health and Tropical Medicine, Tulane University, New Orleans, LA, USA

5 Faculdade de Medicina Hospital São Lucas, PUCRS, Porto Alegre, RS, Brazil

6 Hospital Universitário Walter Cantídio, Universidade Federal do Ceará, Fortaleza, CE, Brazil

7 Faculdade de Medicina, Instituto do Coração, Universidade de São Paulo, São Paulo, SP, Brazil

8 Hospital de Clínicas de Porto Alegre, Universidade Federal do Rio Grande Do Sul, Porto Alegre, RS, Brazil

9 Faculdade de Ciências Farmacêuticas, USP Ribeirão Preto, Ribeirão Prêto, SP, Brazil

Publisher's Note Springer Nature remains neutral with regard to jurisdictional claims in published maps and institutional affiliations. RS 395, Brazil

11 Hospital Universitário Universidade Federal do Maranhão, São Luís, MA, Brazil

12 Universidade Federal de Pernambuco, Recife, PE, Brazil

13 Universidade Federal de Pelotas, Pelotas, RS 96010-610, Brazil

14 Instituto de Medicina Integral Prof Fernando Figueira, Recife, PE, Brazil

15 Universidade Do Estado do Rio de Janeiro, Rio de Janeiro, RJ, Brazil

16 Hospital das Clínicas da Universidade Federal de Minas Gerais, Belo Horizonte, MG, Brazil

17 Hospital das Clínicas de Goiânia, Universidade Federal de Goiás, Goiânia, GO, Brazil

18 Faculdade de Medicina de Botucatu, Botucatu, SP, Brazil

19 Hospital do Coração de Alagoas, Maceió, AL, Brazil

20 Anis Rassi Hospital, Goiânia, GO, Brazil

21 Hospital Universitário Procape, Recife, PE, Brazil

22 Hospital Universitário Antônio Pedro, UFF, Niterói, RJ, Brazil

23 Faculdade de Medicina de Ribeirão Preto, USP Ribeirão Preto, Ribeirão Prêto, SP, Brazil

24 Instituto de Pesquisa, Hospital Israelita Albert Einstein, São Paulo, SP, Brazil 